\title{
LA EXPRESIÓN MUSICAL Y EL CURRÍCULO ESCOLAR
}

\author{
Consuelo Arguedas Quesada
}

\author{
Recibido 13-V-2003 • Aceptado 12-VIII-2003
}

"La sociedad y la familia han de ofrecer a las niñas y a los niños la posibilidad de desarrollarse musicalmente, lo que sin duda va a contribuir en la formación de su personalidad."

(Fridman, 1998 p.113).

\begin{abstract}
Resumen: La educación musical es un arte y un lenguaje de expresión y comunicación que se dirige al ser humano en todas sus dimensiones, desarrollando y cultivando el espíritu, la mente y el cuerpo para llegar a conseguir una educación integral y armónica del individuo. Para las niñas y los niños es sinónimo de juego, movimiento, alegría y emociones, cuya vivencia les permite expresar las sensaciones que les transmite, de manera espontánea y creativa.

La expresión musical brinda beneficios en el área cognoscitiva, desarrolla habilidades, destrezas, hábitos y a la vez estimula la parte afectiva-volitiva de los escolares. Además, está dirigida a todos los estudiantes, no solo para aquellos que poseen talento musical, ya que favorece la libertad, el perfeccionamiento creativo, la actividad y el progreso evolutivo de los escolares mediante el juego. Estos factores permiten el desarrollo global de los escolares y su relación con la vida cotidiana, ya que las experiencias musicales son congruentes con los intereses de los alumnos porque se realizan de manera natural, debido a que la música por sí misma representa algo lúdico para ellos.

Partiendo de estos planteamientos, la educación musical facilita el abordaje de contenidos de otras áreas del currículo escolar, con estrategias metodológicas amenas y creativas.

En el artículo se analizan las características y los objetivos de la educación musical y se mencionan algunos ejemplos de estrategias metodológicas que completan la educación musical con ciencias, español, matemática y estudios sociales.
\end{abstract}

Palabras clave: Expresión Musical, Integración, Currículo Escolar.

\section{Introducción}

El propósito de este artículo consiste en resaltar la importancia de integrar la expresión musical en el currículo escolar. Esta propuesta surge como resultado de la aplicación de la misma, con escolares y estudiantes universitarios de las carreras de Educación Primaria y de Educación Preescolar, de la investigación bibliográfica y del proyecto de investigación ya finalizado "La expresión artística un recurso heurístico para la construcción de procesos pedagógicos desde una perspectiva de género."

Se describen los beneficios, las características y los objetivos de la educación musical, al mismo tiempo que se presenta una reflexión relacionada con la importancia de esta área del conocimiento y su relación con los contenidos curriculares de la escuela.

Hace énfasis en la necesidad de un estímulo sonoro adecuado que favorezca el desarrollo musical de los estudiantes y permita cambios emocionales, cognitivos, sociales y psicomotrices, beneficiando otras áreas curriculares que permitan una visión integral del ser humano. Así por 
ejemplo, los contenidos de español, matemática, ciencias, estudios sociales, inglés, religión, educación física, artes plásticas entre otras, se integran con facilidad con los contenidos de expresión musical, proporcionando estrategias metodológicas interesantes y aprendizajes significativos en los alumnos.

\section{Expresión musical}

La expresión musical tiene como propósito comunicar y transmitir emociones y estados de ánimo por medio de los sonidos. Mediante estímulos sonoros adecuados la música y las artes se interrelacionan y permiten externar la parte subjetiva del individuo de manera creativa y esta es la finalidad de todas las manifestaciones artísticas. "Las artes se interrelacionan, su esencia es una, tienen como finalidad volcar la interioridad humana y compartirla con los demás". (Lavanchi, 1993, p. 58).

Todo lo contrario ocurre si el entorno musical es pobre, ya que se limitan las estrategias metodológicas y las posibilidades de que los aprendizajes sean reveladores en los escolares. Swanwick (1991) considera que existen cuatro modos evolutivos dedicados a la educación musical infantil:

$1 \quad$ El sensorial: ocurre a partir de los tres años de edad y es donde las niñas y los niños responden a los cambios del sonido, sobre todo al timbre y a la intensidad, mediante gestos, movimientos o balbuceos.

1 El manipulativo: se da entre los cuatro y los cinco años y permite un interés en el manejo de instrumentos musicales sencillos.

$1 \quad$ La expresividad personal: se manifiesta de preferencia entre los cuatro y los seis años y se evidencia en el estado anímico de los estudiantes al interpretar las canciones.
1 El modo vernáculo: se considera que aparece durante las primeras fases de producción musical convencional en los estudiantes, al mostrar ideas externas que ellos han captado a partir del canto, al interpretar instrumentos o al escuchar a otras personas; esta fase se manifiesta claramente, entre los siete y los ocho años de edad. "La musicalidad, entendida como la capacidad de percibir, sentir y expresar la música, existe en mayor o menor grado en todas las personas, es necesario desarrollarla y potenciarla." (Bernal, 1999, p. 14).

De igual manera, estas etapas evolutivas forman parte del desarrollo integral del individuo y por consiguiente se manifiestan no sólo en la música, sino en las otras áreas curriculares escolares; por lo tanto, merecen la atención de los docentes en los centros educativos. Como consecuencia, es importante integrar la expresión musical en el proceso educativo escolar para propiciar aprendizajes dinámicos y novedosos.

En la expresión musical, al igual que en el proceso de comunicación, es necesario que los estudiantes transiten en la etapa de exploración, donde se realizan aprendizajes por descubrimiento mediante la acción; la de imitación, donde se repiten diferentes modelos; la etapa de improvisación donde se ensayan y se combinan espontáneamente diferentes elementos musicales y por último la etapa de creación, en la que se plasman esquemas adquiridos $\mathrm{u}$ originales, producto de una inspiración.

La educación musical parte de las experiencias anteriores realizadas en el hogar y se fundamentan en las necesidades e intereses de los estudiantes.

Para brindar oportunidad de desarrollar la expresividad y la capacidad de producir música, de vivirla, de apreciarla y disfrutarla, la actividad musical debe ser espontánea y fundamentada en el juego. Utilizando actividades lúdicas, el ritmo, por ejemplo, se favorecen las posibilidades 
motoras que el niño y la niña traen desde su nacimiento, permitiendo el desarrollo de la memoria y la atención. Por medio de la audición, se capacita a los escolares para que perciban y seleccionen intensidades, velocidades, timbres sonoros y alturas de los sonidos. De igual forma, la apreciación musical estimula la sensibilidad auditiva, el respeto hacia sí mismo y hacia los demás y el sentido crítico en los oyentes.

\subsection{Beneficios de la educación musical}

Frega (1998), considera que para lograr los objetivos musicales en la escuela, es conveniente realizar de manera paulatina actividades auditivas, rítmicas, expresivas, de creación e interpretación que pueden desarrollarse en las siguientes áreas de trabajo:

Área cognoscitiva:

1. La música y el desarrollo histórico del hombre.

2. La música y la sociedad contemporánea.

3. La forma musical.

4. La música y las otras áreas del currículo escolar.

Es importante recalcar que la música ha acompañado al ser humano desde el inicio de su existencia, en la guerra, en las actividades de labranza, en la cacería, durante los ritos festivos y en los momentos fúnebres. De igual manera está unida al folklore de los pueblos y a sus efemérides; sin dejar de lado los ritmos y melodías contemporáneas que son una muestra del desarrollo y a la vez de la problemática del mundo en que vivimos. Así por ejemplo el carácter subjetivo de una obra musical del periodo romántico contrasta con la rigurosidad de una obra clásica apegada a las normas rígidas de su estructura formal, o aún más, a una obra impresionista donde los efectos sonoros y rítmicos pretenden "dar la impresión" de lo que el autor desea expresar. Estas características musicales están en total relación con el momento histórico del hombre y por lo tanto reflejan su acontecer cotidiano. Es así como crea diversas manifestaciones sonoras y las clasifica teniendo en cuenta sus características específicas y su forma musical; por ejemplo se pueden citar los conciertos, los valses, las sinfonías, la ópera, la música de cámara, entre otras muchas posibilidades.

En la apreciación musical y la expresión corporal de los escolares muchas de ellas son de gran utilidad, al igual que otras más sencillas en su composición como la canción, el canon ${ }^{1}$, el ostinatti ${ }^{2}$, el eco melódico y rítmico ${ }^{3}$, las polirritmias ${ }^{4}$ y los quolibets ${ }^{5}$.

Otro componente importante en el desarrollo musical de los estudiantes es la lecto-escritura musical, que corresponde a los procesos de enseñanza y aprendizaje de leer y escribir o graficar la música. Se aconseja realizarlos no sólo en forma teórica sino más bien, mediante vivencias de los elementos musicales en forma corporal, con instrumentos o con materiales auxiliares; es decir se debe interiorizar primero, para una mejor comprensión teórica.

A partir de estas estrategias metodológicas musicales, las otras áreas del currículo escolar se pueden integrar al trabajo de aula para enriquecerse y desarrollarse en forma amena y significativa para las niñas y los niños.

\footnotetext{
"La música en la educación general no apunta a la formación de especialistas en un área dada, sino a la promoción del desarrollo pleno de las facultades totales del hombre siempre en orden al aprovechamiento personal y colectivo de las potencialidades individuales.” (Frega, 1998, p. 20).
}

Área de habilidades, destrezas y hábitos

1. El canto en la escuela.

2. Ejecución instrumental percusiva o con instrumentos melódicos sencillos 
que se realiza en forma individual o colectiva.

\section{Educación auditiva.}

\section{Educación rítmica.}

El canto es el punto de partida de toda actividad musical porque con él se estimula el sentido rítmico y melódico, el oído, el aparato respiratorio, la articulación, la emisión correcta de las palabras, la vivencia de distintas velocidades e intensidades. Favorece además la memoria, el desarrollo del lenguaje oral y la entonación, ayuda en la socialización de los estudiantes y a liberar emociones y sentimientos.

Recordemos que las primeras formas de comunicarse el bebé con su madre son los balbuceos, las sonrisas o el llanto. Aún más, ya desde la prehistoria, el hombre primitivo cantaba en sus momentos de gloria y de tristeza, para relacionarse con los demás y alabar a sus dioses.

La canción complementa cualquier actividad escolar al brindar múltiples posibilidades didácticas, es por este motivo que se debe utilizar con frecuencia durante el proceso educativo. La selección de las canciones debe estar de acuerdo con los intereses de los estudiantes, el vocabulario debe ser adecuado a la edad, al igual que el ritmo y los sonidos utilizados. Así mismo las canciones que se entonen se recomienda que abarquen diferentes temáticas: populares, patrióticas, recreativas, típicas, folklóricas, entre otras. "Cantar es continuación del hablar, por lo que es muy importante elegir buenos textos para las canciones". (Pascual, 2002, p. 241).

El canto se puede trabajar con acompañamiento corporal o con acompañamiento instrumental, cantar en canon, cantar todos al mismo tiempo o al unísono, cantar con movimientos que expresan lo que sugiere la letra, canto en grupo con un solista o guardar la canción, es decir cantar cuando el maestro lo indica y cuan- do no lo haga los participantes lo siguen realizando mentalmente.

La práctica instrumental implica tocar, ver y hacer, con lo cual se realiza un intercambio entre la audición, la motricidad y la vista. Desarrolla capacidades musicales, psicomotoras y corporales, contribuye con la agudeza auditiva para intervenir en el momento correcto y a la vez diagnostica problemas motores, respiratorios o auditivos. Desarrolla la sensibilidad y cultiva valores espirituales, favoreciendo el trabajo grupal y la responsabilidad.

La educación auditiva se relaciona con contenidos del lenguaje y de las ciencias: como por ejemplo el sonido y el silencio, sonidos onomatopéyicos y del ambiente, sonidos de diferente duración, sonidos con distinta velocidad e intensidad o con diferente timbre. También se puede establecer la diferencia entre lo que es sonido y ruido; entendiendo el primero como aquel que se produce por vibraciones regulares y armónicas, mientras que el ruido es producido por vibraciones irregulares. "Ruido es cualquier señal sonora indeseada" (Shafer, 1969, p. 29). La música contemporánea utiliza ambos, es decir, ruidos y sonidos para realizar las composiciones; la grafía musical empleada es similar al garabateo infantil, pues no corresponde a la grafía convencional.

El sentido del ritmo es desarrollado por el ser humano según sus experiencias y sus posibilidades, por esta razón es importante estimular la educación rítmica, ya que por medio de ella se favorecen la coordinación motriz y las funciones corporales en forma armoniosa con el tiempo y el espacio; de igual manera, se estimulan la concentración, la memoria y la atención.

La educación rítmica comprende: el pulso, que es la constante, el "tempo" o latido regular de la música; el acento, que son los pulsos fuertes o más acentuados; y el ritmo de frase que corresponde a los sonidos cortos, sonidos largos o ausencia del sonido, es decir a las subdivisiones de los tiempos de la música con sus respectivos silencios. 
Área afectivo-volitiva:

1. La música representa un medio de autoexpresión.

2. Se desarrolla la sensibilización por medio de la apreciación musical.

3. Fomenta valores de amor, libertad, belleza, respeto y verdad, entre otros.

\section{Estimula el proceso creativo.}

Al proporcionar obras musicales diferentes como el vals, la sonata, el concierto, la sinfonía, entre otras, todas ellas de distintos periodos y compositores, se desarrolla la sensibilidad estética y el gusto artístico, lo que permite a los estudiantes captar no sólo su mundo exterior sino también su mundo interior. Es por esta razón que la apreciación musical es muy importante aplicarla en la educación primaria, porque "la música tiene una gran capacidad para conmover de forma casi universal a todos los individuos en función de los parámetros musicales que establece cada comunidad o cultura; y es al mismo tiempo, una vía de expresión emotiva”. (Pascual, 2002, p. 301).

En la audición intervienen aspectos afectivos, cognitivos y psicológicos que influyen en la interpretación de la misma. No obstante que la apreciación musical permite la comparación, la selección, el análisis y la síntesis, la percepción del estímulo musical es global, formando una totalidad sonora.

Oír y escuchar música son actividades diferentes; el primero implica tener atento el canal auditivo, pero no la parte de las emociones; se oye en forma involuntaria, sin sentir el estímulo sonoro. Para escuchar se necesita la concentración, las respuestas físicas, afectivas e intelectuales, que la música le sugiere al ser humano.

La apreciación musical trae como consecuencia el desarrollo de la sensibilidad y de la auto-expresión, fomentando a la vez el proceso creativo. Esto se logrará programando cuidadosamente y con anticipación el repertorio musical a utilizar, teniendo en cuenta las necesidades e intereses de los estudiantes.

\subsection{Características de la educación musical}

Pascual (2002), menciona los siguientes aspectos sobresalientes de la educación musical:

Valor educativo de la música

La finalidad es hacer música de una manera integral, teniendo en cuenta las áreas sociológicas, psicológicas, psicomotoras e intelectuales.

La educación musical es para todos

No está dirigida sólo a personas con talento musical, no se trata de hacer músicos sino personas que aprecien, valoren y amen la música.

\section{Libertad y creatividad}

En la educación musical es muy importante la espontaneidad, los resultados no son lo primordial, sino el proceso y la participación de los individuos; algunas veces la creación se basa en imitaciones, sin embargo, los estudiantes aplican sus percepciones y las capacidades propias en busca de su originalidad.

La creatividad es una transversalidad indispensable en todo el proceso de enseñanza-aprendizaje ya que define el área artística del individuo. La creación personal será el resultado de la motivación y del constante desarrollo de habilidades, destrezas y hábitos. 
Progresión

La educación musical debe acompañar al niño y a la niña en todo el proceso evolutivo, adaptándose a sus intereses y capacidades según la edad.

\section{Actividad}

Está basada en la experimentación y en la participación; aprender música es hacer música por medio de un lenguaje vivo, donde el alumno y la alumna son los verdaderos protagonistas, y el docente es quien guía el proceso de aprendizaje.

\section{Variedad}

Para lograr una educación musical adecuada y placentera, es necesario incluir, dentro del proceso educativo, los diferentes aspectos que la comprende.

\section{Actividad lúdica}

Una manera muy acertada de vivenciar la música es mediante el juego, con objetivos claramente definidos. De esta manera, los aspectos teóricos se interiorizan en forma corporal, utilizando instrumentos percusivos o melódicos, así como también integrando materiales auxiliares.

\section{Global}

Se relaciona con la expresión literaria, la plástica, la expresión dramática, estudios sociales, ciencias, matemática y en general, con el desarrollo integral de los escolares.

\section{Impregna en la vida cotidiana}

La educación musical en la escuela pretende propagarse a la familia y a la comunidad, de manera que se establezca un vínculo social, afectivo y cognoscitivo.

\subsection{Objetivos de la educación musical}

Teniendo en cuenta los beneficios y las características antes mencionadas, se pueden sintetizar los objetivos de la Educación Musical de la siguiente manera:

Contribuir al desarrollo pleno del sujeto, posibilitándole vivencias estético musicales integradas al proceso educativo.

1 Desarrollar las habilidades musicales de las alumnas y los alumnos por medio de experiencias variadas y seleccionadas.

$1 \quad$ Motivar a las estudiantes y los estudiantes hacia la apreciación y el gusto por la música, como un medio de expresión del ser humano.

$1 \quad$ Desarrollar la sensibilización musical ante diversos estímulos sonoros.

Cuidar y enriquecer el entorno sonoro.

Favorecer la creatividad de las estudiantes y los estudiantes mediante recursos sonoros variados.

Estimular el trabajo individual y grupal a través de actividades musicales de apreciación musical, rítmicas, auditivas, de ejecución instrumental o vocal, que favorezcan el desarrollo pleno del individuo.

\subsection{La expresión musical integrada} al aula escolar

La percepción, la expresión y la comunicación son capacidades que se desarrollan en la etapa escolar en el ámbito psicomotor, emocional y cognitivo. 
De igual forma, en la educación musical se pretende desarrollarlas en los estudiantes poniendo en práctica actividades que les favorezcan como intérpretes, auditores y receptores. Asimismo, los estimula como personas creativas, capaces de expresar vivencias, sentimientos y emociones, favoreciendo la autoestima y la autoimagen. Es decir, utiliza lenguajes que facilitan la comunicación con los estudiantes mismos, con los demás y con los objetos que los rodean.

La expresión musical desempeña un papel de interacción social y afectiva que se realiza por medio de los cantos, juegos y rondas tradicionales de una región. Debemos recordar que hoy día, en muchos centros educativos, la población estudiantil abarca estudiantes de otras nacionalidades y gracias al aporte musical se comparten aspectos culturales entre los escolares, favoreciendo además la amistad, el respeto y el diálogo.

La integración curricular escolar se beneficia también con la música, al propiciar estrategias metodológicas que promueven aprendizajes significativos. Por este motivo, las actividades musicales permiten realizarse en forma global, es decir, además de los contenidos específicos, pueden relacionarse con otros temas de estudio; en consecuencia, se convierten en un instrumento útil para el resto de los aprendizajes no musicales.

Para los escolares la música representa una atracción, una posibilidad de entretenimiento y de expresión individual y colectiva. Para los educadores es una herramienta que facilita la motricidad, el lenguaje, la afectividad, el desarrollo rítmico y la coordinación motriz; conveniente como elemento terapéutico, como relajante o motivador de creaciones. De igual manera, la expresión musical propicia sesiones activas donde los estudiantes "vivencian" los temas por tratar, disfrutan el aprendizaje, gozan de las creaciones propias y las de los demás; representan experiencias importantes para ellos, al dejar de ser tan solo datos memorísticos que se estudian para realizar un examen. "En la expresión musical se pretende que niños y niñas adquieran una progresiva capacidad para servirse de este procedimiento de comunicación y representación al servicio de los objetivos educativos generales.” (Bernal, 1999, p. 10).

La vivencia de experiencias repercute favorablemente en el quehacer educativo, ya que al estar de acuerdo con los intereses infantiles, los escolares consideran el aprendizaje como algo natural y espontáneo; es decir, la música en sí misma representa un elemento lúdico para ellos.

Es necesario recordar, que los juegos didácticos en ningún momento deben desvirtuar la esencia de aquello que queremos sea vivenciado por los escolares y posteriormente sea comprendido. Las actividades musicales lúdicas, son estrategias metodológicas que favorecen el aprendizaje que se desea trasmitir; no se realizan por el simple hecho de jugar por jugar.

Cabe mencionar que la educación musical no es privilegio de unos pocos, la musicalidad está presente en todas las personas, en mayor o menor grado, y es indispensable potenciarla, sin excepción. Al respecto Amparo Porta (1999), considera que el fenómeno sonoro forma parte de la vida de los niños y de las niñas y por lo tanto, tienen todo el derecho de experimentar y jugar con él, trayendo como consecuencia el disfrute y el conocimiento del mismo.

Algunas veces, los aprendizajes se vuelven repetitivos y áridos, olvidándose que los estudiantes son seres completos, con sus intereses y sus experiencias; por esta razón, se recomienda que los educadores ofrezcan a los alumnos posibilidades para descubrir en la educación musical un medio de expresión maravilloso, cuya organización de sonidos tiene como fin ser escuchada y apreciada, integrándose además a la enseñanza de otras materias escolares.

Con el fin de ilustrar la integración de la expresión musical en el currículo de la escuela, se sugieren algunas estrategias 
metodológicas para las áreas de ciencias, matemática, español y estudios sociales. En ellas se rescatan aspectos específicos de educación musical, pero a la vez se enfatiza en contenidos de las materias mencionadas.

\section{Ciencias}

Eje temático: los seres humanos somos parte integrante de la naturaleza.

Contenido: diferentes ambientes donde se desarrollan los seres vivos.

\section{Estrategias metodológicas:}

- Se escucha una obra musical que presente alguna característica musical específica que permita identificar por ejemplo:

1 Cambios de intensidad que comprendan sonidos fuertes, suaves, muy fuertes o muy suaves.

1 Cambios de velocidad que incluyan rápido, lento, muy rápido o muy lento.

1 Sonidos agudos, graves o repetidos; es decir diferentes alturas del sonido.

1 Timbre sonoro o sonido que caracteriza un instrumento musical, una voz o una acción.

A continuación se pregunta a los estudiantes si al escuchar la obra musical les causó sensaciones de alegría, tristeza, enojo, nostalgia, paz, recuerdos de algún lugar en especial, algún animal o situación vivida.

- Como consecuencia de las impresiones expresadas, se comenta sobre los posibles hábitats o lugares donde viven distintos organismos y que les ofrece las condiciones para realizar sus funciones vitales, que podrían estar reflejados en la obra musical escuchada, teniendo en cuenta las características sonoras específicas.

- Los alumnos emiten sonidos onomatopéyicos con la boca o con cualquier otra parte del cuerpo, simulando los timbres sonoros producidos por los animales que habitan dicho ecosistema.

- Los estudiantes hacen sonidos con instrumentos de percusión, elaborados con materiales desechables o con elementos de la naturaleza, con la finalidad de ayudar en la creación del ambiente sonoro del lugar.

Español

Eje temático: Participación activa en el uso del lenguaje oral y escrito.

Contenido: lectura y escritura de textos sencillos.

\section{Estrategias metodológicas:}

Los estudiantes elaboran un texto donde se describan animales, plantas y seres inertes de un hábitat específico.

- Cada niña y niño improvisa una melodía sencilla que le adjunta al texto creado, de esta manera el texto se convierte en canción.

- Los alumnos cantan la canción creada en forma individual y el docente las recopila utilizando la grabación.

- $\quad$ El maestro selecciona algunas de las canciones creadas por los escolares y se procede al aprendizaje de ellas de 
manera grupal. A continuación se sugieren las pautas para llevar a cabo el aprendizaje de una canción:

1 Práctica del eco melódico donde la persona que guía la actividad canta una frase musical, los estudiantes la escuchan y posteriormente la entonan.

Posteriormente se practica el canto de pregunta-respuesta. En esta actividad la persona que está guiando el proceso canta una frase musical y el resto de los participantes entona la siguiente frase, como si se tratara de una conversación.

$1 \quad$ Cambio del guía en la práctica del eco melódico, es decir, inician la actividad los estudiantes.

Cambio del guía en la práctica de la pregunta-respuesta; en este caso empiezan la actividad los escolares.

$1 \quad$ Se realiza el canto total o canto completo, interpretado por los estudiantes sin la ayuda del docente.

\section{Estudios Sociales}

Eje temático: Peculiaridades culturales del distrito, del cantón y de la provincia.

Contenido: Distinción de las principales características culturales de la provincia.

\section{Estrategias metodológicas:}

Comentar aspectos culturales sobresalientes de la provincia: costumbres, tradiciones, festividades, comidas, juegos, poesía, actividades artísticas y otras, teniendo en cuenta la nacionalidad de todos los estudiantes.

- A partir de rimas y trabalenguas que reflejen características del lugar los estudiantes percuten el pulso o constante musical, también conocido como el "siempre igual"; el acento o pulso acentuado; o el ritmo que comprende sonidos largos, cortos o ausencia del sonido. Utilizar percusiones corporales o instrumentos de percusión.

- Bailar alguna danza representativa del lugar.

- Realizar juegos o actividades donde se interpreten cantos y que ofrezcan el intercambio cultural; de esta manera se integra a los escolares de diferentes nacionalidades que puedan encontrarse dentro del grupo.

\section{Matemática}

Eje temático: El ser humano reconoce las líneas y los planos y entra en contacto con ellos de manera objetiva.

Contenido: Elementos geométricos. Líneas rectas.

\section{Estrategias metodológicas:}

Llevar a cabo comentarios relacionados con diferentes clases de líneas, como por ejemplo: horizontal, vertical, inclinada. ¿Cómo son? ¿En qué lugares de la clase se encuentra alguna de ellas?

- Haciendo uso de cintas de tela (anteriormente las han utilizado para improvisar movimientos en forma libre), los escolares las colocan en el piso de la clase en forma horizontal, vertical o inclinada. 
- $\quad$ Se les indica la siguiente consigna:

$1 \quad \mathrm{Al}$ escuchar un sonido grave en forma consecutiva, se desplazan al lado de la cinta los estudiantes que la hayan colocado en posición horizontal.

$1 \quad$ Al escuchar un sonido agudo consecutivo, se desplazan al lado de la cinta, los estudiantes que la hayan colocado en posición vertical.

$1 \quad \mathrm{Al}$ escuchar una serie de sonidos en forma descendente (de agudo a grave), se desplazan al lado de la cinta los estudiantes que la hayan colocado en posición inclinada Los sonidos se ejecutan con flauta dulce, guitarra, organeta, piano, o cualquier instrumento melódico.

Se debe tener presente que es importante la vivencia y la discriminación anterior de sonidos agudos y graves.

Con esta actividad se pretende establecer además del desarrollo auditivo, una relación entre el estímulo sonoro y la posición de las líneas.

Contenido: Figuras planas.

Una variante de la estrategia anterior puede ser la siguiente:

- Se realizan comentarios acerca de algunas figuras planas: rectángulo, círculo, triángulo. ¿Cómo son? ¿En qué lugares de la clase se encuentran algunas de ellas?

- Los escolares colocan las cintas en el piso de la clase formando, círculos rectángulos o triángulos.

- $\quad$ Se propone la siguiente consigna:
$1 \quad \mathrm{Al}$ escuchar el sonido del pandero, (instrumento de percusión de sonido indeterminado que tiene una membrana o parche: se diferencia de la pandereta porque no tiene chapas), se desplazan los estudiantes que colocaron su cinta en forma de círculo. Si no se cuenta con un pandero en la actividad propuesta, puede ser sustituido por la pandereta.

$1 \quad \mathrm{Al}$ escuchar el sonido del triángulo, se desplazan los estudiantes que colocaron su cinta en forma de triángulo.

$1 \quad$ Al escuchar el sonido de la caja china, (instrumento de percusión de sonido indeterminado, formada por un trozo de madera con un orificio que le sirve de caja de resonancia), se desplazan los estudiantes que colocaron su cinta en forma de cuadrado.

Es importante recordar que los escolares deben discriminar con antelación los distintos timbres sonoros.

Con esta actividad se establece además del desarrollo auditivo, se reafirman los conceptos del círculo, el triángulo y el rectángulo estableciendo además, la relación de las figuras geométricas mencionadas y la forma específica del pandero, la caja china y el triángulo.

\section{A manera de conclusión}

La educación musical es un lenguaje que le permite al ser humano comunicarse y expresarse en todas sus dimensiones, desarrollando su espíritu, su mente y su cuerpo, con la finalidad de lograr una educación integral y armónica. Además, para las niñas y los niños escolares representa 
una actividad lúdica divertida que facilita la posibilidad de comunicar emociones y sentimientos en forma espontánea. Proporciona mejores relaciones entre los estudiantes, se refuerza la autoestima y la seguridad personal, ofrece la posibilidad de estimular la sensibilidad y la creatividad, además permite implementar en los estudiantes el sentido crítico y el respeto hacia los demás.

De esta manera, los escolares perciben el entorno sonoro como parte de su medio y se convierten en auditores, intérpretes y creadores manifestando sus experiencias personales, sus pensamientos, las fantasías y las vivencias lúdicas mediante diversas actividades musicales que les facilitan el desarrollo de la voz, el oído musical, el movimiento y las destrezas en la ejecución instrumental.

La educación primaria a su vez, busca impulsar la elaboración de juicios personales creativos, basados en un adecuado equilibrio del conocimiento y de la afectividad, características que se interrelacionan con la labor de la educación musical, ya que ésta pretende ofrecer estrategias individuales y colectivas que benefician las relaciones interpersonales e intrapersonales, que favorecen el desarrollo de valores y actitudes entre los estudiantes. Al mismo tiempo, se preocupa por utilizar de manera eficaz y funcional, aprendizajes que utilicen diferentes tipos de lenguajes para interpretarlos de forma crítica, novedosa y activa integrando estrategias relacionadas con otras áreas artísticas como la plástica, la literatura y la expresión dramática. Es así como se realizan experiencias afectivas, sociales, motrices y cognoscitivas que le permiten a los escolares identificarse y participar en su entorno social en forma individual y grupal.

No obstante, existe el criterio equivocado de algunos docentes de menospreciar el valor formativo e interdisciplinario de la educación musical y ellos ofrecen sesiones de esta disciplina pobres en contenidos, repetitivas y de poca atracción para los estudiantes; o aún peor, ni siquiera se imparte en los centros educativos por considerarse innecesaria y hasta una pérdida de tiempo.

Con este artículo pretendo por el contrario, hacer conciencia de la importancia de integrar la educación musical con las distintas disciplinas del currículo escolar, ya que constituye una fusión perfecta para el crecimiento integral del ser humano conformando un eje integrador imprescindible, capaz de propiciar estrategias metodológicas y aprendizajes diferentes y agradables para los estudiantes y los docentes de educación primaria.

\section{Notas}

1. Dos o más grupos ejecutan en forma sucesiva una misma melodía o esquema rítmico sonoro.

2. Esquema rítmico o melódico que se repite siempre igual durante la ejecución de la obra musical.

3. Esquema rítmico o melódico que se repite de igual forma, después de ser escuchado anteriormente.

4. Varios ritmos diferentes que se ejecutan al mismo tiempo.

5. Dos o tres melodías diferentes que se ejecutan en forma simultánea.

\section{Referencias bibliográficas}

Beuchat R., Cecilia. Desarrollo de la Expresión Integrada: Club Cli-Clo-Pips. Chile: Editorial Andrés Bello, 1993.

Eufonía 8. Creatividad-Improvisación. Didáctica de la Música. España: Editorial GRAÓ, 1997.

Eufonía 7. Procedimientos en la Educación Musical. Didáctica de la Música. Barcelona: Editorial GRAÓ, 1997. 
Eufonía 14. La Música en la Educación Infantil. Didáctica de la Música. Barcelona: Editorial GRAÓ, 1999.

Frega, Ana Lucía. Música para Maestros. España: Editorial GRAÓ, 1998.

Fridmann, R. El nacimiento de la inteligencia musical. Buenos Aires: Editorial Guadalupe, 1988.

Pascual Mejía, Pilar. Didáctica de la Música. Madrid: Pearson Educación, 2002.

Programa de Estudios Ciencias. Primer Ciclo de Educación General Básica. San José: Ministerio de Educación Pública, 2001.

Programa de Estudios de Español. Primer Ciclo de Educación General Básica. San José: Ministerio de Educación Pública, 2001.
Programa de Estudios Sociales. Primer Ciclo de Educación General Básica. San José: Ministerio de Educación Pública, 2001.

Swanwick, K. Música, Pensamiento y Educación. España: Morata 1991.

Shafer R. Murray. El nuevo paisaje sonoro. Canadá: Editorial Ricordi Americana, 1969.

Sefchovich, Galia-Waisburd, Gilda. Expresión Corporal y Creatividad. México: Editorial Trillas, 1997.

Vargas D., Ana Isabel. Técnicas para el Desarrollo de la Expresión y el Movimiento en la Escuela Primaria o Básica. Módulo 7 Unidad 7,3. San José: Oficina Subregional de Educación de la UNESCO para Centroamérica y Panamá.

Consuelo Arguedas Quesada Investigadora del Instituto de Investigaciones para el mejoramiento de la Educación Costarricense (IIMEC) 